\title{
Relation between space charge limited current and power loss in open drift tubes
}

\author{
Debabrata Biswas and Raghwendra Kumar \\ Theoretical Physics Division, Bhabha Atomic Research Centre, Mumbai 400 085, INDIA *
}

(Dated: July 24, 2021)

\begin{abstract}
Drift space is a region free from externally applied fields. It is an important part of many devices involving charged particle beams. The space charge effect imposes a limit on the current that can be transported through a drift space. A reasonable estimate of the space charge limited current density $\left(J_{S C L}^{c}\right)$ in closed drift tubes can be obtained from electrostatic considerations despite the fact that charge particle transport results in electromagnetic radiation. We deal here with the situation where the drift tube is open to electromagnetic radiation, for instance due to the presence of a dielectric window. In such cases, electromagnetic radiation leaks out of the window which results in a decrease in the average kinetic energy of electrons. If the injected current density is much lower than $J_{S C L}^{c}$, power loss does not result in a change in the transmitted current. As the injected current density is increased, power loss increases and at a critical value lower than $J_{S C L}^{c}$, reflection of electrons begins to occur. We also show that the lowering of the space charge limited current on account of power loss can be incorporated in the standard electrostatic picture by introducing an effective voltage for the collection plate.
\end{abstract}

PACS numbers: 52.59.Mv, 52.59.Sa, 85.45.-w

\section{INTRODUCTION}

The motion of charged particles in a drift space is of considerable interest in fields such as high power microwaves and accelerators [1, 2]. Here, the mutual interaction among the charged particles (known as the space charge effect) plays an important role in the dynamics of an intense beam in such cases [2]. If a beam of charged particles is injected into a drift region, space charge forces oppose the incoming beam. For a low intensity beam, space charge effect is negligible and the transmitted current is equal to the injected current. As the injected current increases to a certain critical value (commonly referred to as the space charge limited (SCL) current), a virtual cathode appears in the drift space from which some of the electrons turn back to the injecting plane [2, 3]. As the injected current is increased beyond the SCL value, the transmitted current decreases and saturates asymptotically to a much smaller value when the injection energies are non-relativistic.

It is possible to estimate the space charge limited current for a non-relativistic electron beam moving in an infinite parallel plate drift space. The SCL current density is given by [2, 3]

$$
J_{\mathrm{SCL}}^{c}=\frac{32}{9} \epsilon_{0} \frac{V^{3 / 2}}{L^{2}}\left(\frac{2 e}{m_{0}}\right)^{1 / 2}
$$

with the injection velocity $v_{0}=\sqrt{2 e V / m_{0}}$ and $L$ being the separation between the plates. Here $e$ and $m_{0}$ are the electron charge and rest mass respectively. On the other hand, for a relativistic solid beam of radius $r_{b}$ in an infinitely long drift tube of radius $R$, a reasonable approximation is 4

*Electronic address: dbiswas@barc.ernet.in, raghav@barc.ernet.in

$$
J_{\mathrm{SCL}}^{c} \simeq m_{0} c^{3} \frac{4 \epsilon_{0}}{e}\left[r_{b}^{2}\left[1+2 \ln \left(R / r_{b}\right)\right]\right]^{-1}\left(\gamma_{0}^{2 / 3}-1\right)^{3 / 2}
$$

where $\gamma_{0}$ is the relativistic factor for the injected velocity.

When the injected current density $J_{I N}$ exceeds $J_{\mathrm{SCL}}^{c}$, the transmitted current density $J_{T R} \leq J_{\text {SCL }}^{c}$ and its approximate analytical value can be obtained from the so called classical theory in the 1-dimensional case [2, 3].

It should be noted that the above estimates for the space charge limited current in a drift tube are derived from electrostatic models. In reality, the mutually interacting charged particles radiate and in the process, lose their kinetic energy to the fields. In closed drift tubes, the electrostatic predictions are nevertheless good as the radiated electromagnetic energy is reabsorbed by the electrons. However, when a drift tube is open to electromagnetic radiation, this energy leaks out as we shall demonstrate here. This leads to a drop in the kinetic energy of the transported electrons. When the injected current density, $J_{I N}<<J_{S C L}^{c}$, the transmitted current $J_{T R}$ is unaffected by the slowing down of electrons as the charge density increases to compensate for the lower velocity. However as $J_{I N}$ increases further, reflection begins to occur at a value (of $J_{I N}$ ) smaller than $J_{S C L}^{c}$. We shall show here that the lowering of $J_{S C L}^{c}$ can be predicted reasonably well within the electrostatic framework by altering the boundary conditions to account for power loss.

It may be noted that a similar phenomenon occurs in open diodes. It has recently been shown [5] that leakage of electromagnetic energy from a diode results in lower transmitted current. Furthermore, the electromagnetic power loss can be incorporated into the standard Child-Langmuir 6, 7] expression through an effective voltage. For a given applied potential difference and anode-cathode separation, the modified Child-Langmuir law can also be used to predict the maximum power that can leak from an open-diode [5].

In this paper, we present results of particle-in-cell 


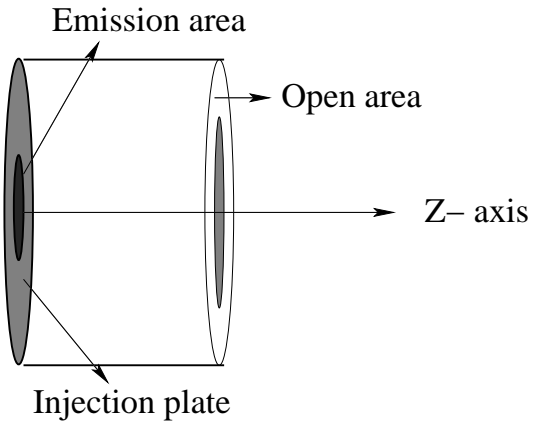

(a)

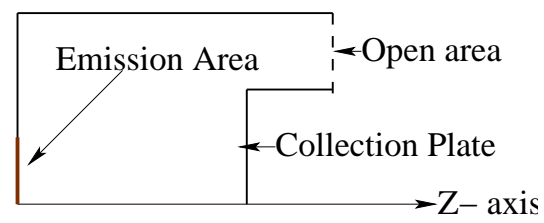

(b)

FIG. 1: Schematic of the open drift tube and the simulation geometry as used in the PIC code XOOPIC.

(PIC) simulation for open drift tubes. In section \1 we demonstrate that an opening in the drift tube allows radiation to leak out and lowers the kinetic energy of electrons. We next show that the critical current density at which reflection begins to occur in open drift tubes $\left(J_{S C L}^{O}\right)$ is lower as compared to closed drift tubes. We also show that the transmitted current decreases as the injected current is increased beyond $J_{S C L}^{O}$. In section III we propose that the power loss in open drift tubes can be incorporated within the electrostatic framework by introducing an effective voltage for the collection plate. The predictions of this effective energy theory is then compared with PIC simulations. Finally, the significance of this study and a summary of our results are presented in the concluding section.

\section{POWER LOSS IN OPEN DRIFT TUBES}

We shall first demonstrate that an opening in the drift tube leads to power loss in the form of electromagnetic radiation and hence to a lowering of the kinetic energy of electrons reaching the far end of the drift tube (hereafter referred to as the collection plate).

The drift tube under consideration is a metallic cylindrical cavity with one end partially open (see fig 1). The radius of the collection plate $\left(R_{C P}\right)$ is varied in the simulation. The electrons are injected from the injection plate in the axial direction with uniform velocity. The radius $R_{1}$ of the drift tube is taken to be $12.5 \mathrm{~cm}$ while the length $L$ is $2.5 \mathrm{~cm}$. An electron beam of radius $3.5 \mathrm{~cm}$ is used with an injection energy of $50 \mathrm{KeV}$.

The results presented here have been obtained using the fully electromagnetic two and half dimensional PIC code XOOPIC [8]. The cavity wall is considered as "Con-

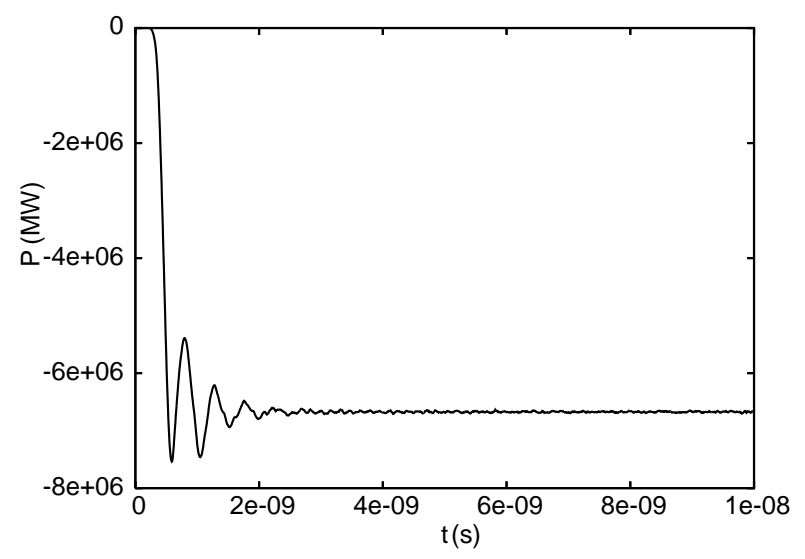

FIG. 2: The power radiated through the open area is computed using the Poynting vector. The equilibrium value is found to be $6670 \mathrm{KW}$.

ductor" in the simulation; the emission model is chosen to be "VarWeightBeamEmitter" while the open window is modelled by an "Exitport". The number of mesh points in the radial and the axial directions is typically 64 and 128 respectively. The time step is typically taken to be $4 \times 10^{-13}$ s. Note that there is no externally applied magnetic field in the simulation.

For the injection energy and plate separation under consideration, Eq. 1 predicts the space charge limited current $I_{S C L}^{c}=1283$ amperes. While this is strictly true for infinite parallel plates separated by a distance $L$, for the closed cylindrical drift tube geometry $\left(R_{C P}=\right.$ $12.5 \mathrm{~cm}$ ), our PIC simulations show that $I_{S C L} \simeq 1320$. Hereafter, we shall refer to this value as $I_{S C L}^{c}(2)$ where the superscript $c$ refers to the closed drift tube and (2) refers to the 2-dimensional case.

In order to demonstrate that charge particle transport indeed results in electromagnetic radiation which can leak out from an open drift tube, we have carried out a simulation with the radius of the collection plate $R_{C P}=8 \mathrm{~cm}$ and the injected current $I_{I N}=500 \mathrm{~A}$. The electromagnetic power emitted from the open area is plotted in fig. 2. In order to account for this power loss, we also plot the energy distribution of the electrons reaching the collection plate in fig. 3 .

As reflection of electrons does not occur at this value of injected current, the ratio $e P / I_{T R} \simeq e 6670 / 500=$ $13.34 \mathrm{KeV}$ is the average energy loss that a single electron suffers. In other words, the average kinetic energy of electrons reaching the collection plate should be 36.66 $\mathrm{KeV}$. This should reflect in the energy distribution of electrons at the collection plate. Indeed Fig. 3] shows that the kinetic energy peaks at $36.9 \mathrm{KeV}$ which agrees well with the value expected from energy conservation.

It is thus clear that electrons lose energy in the form of electromagnetic radiation and as a result slow down. This leads to an increase in charge density and hence enhances the space charge effect. For the values of injected current, energy and power loss in the above example, the space charge effect is not large enough to cause reflection of electrons. As the injected current is increased fur- 


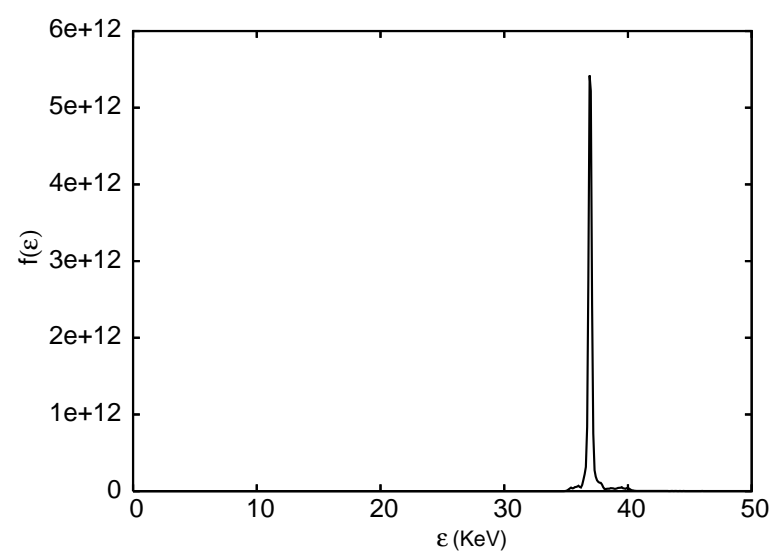

FIG. 3: The kinetic energy distribution of the electrons reaching the collecting plate. There is a sharp peak at $36.9 \mathrm{KeV}$.

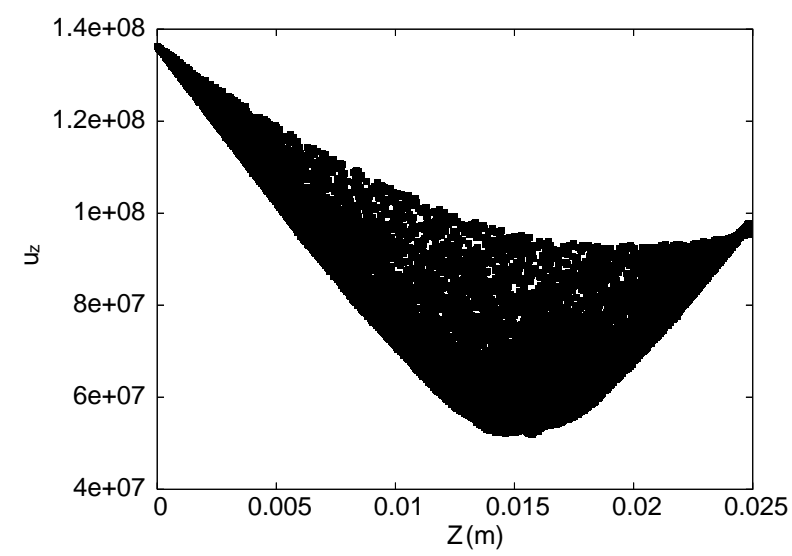

FIG. 4: The phase space for $I_{I N}=910$ A. There is no reflection at this injected current.

ther, power loss increases as well thereby leading to an increase in charge density. For the open drift tube under consideration, reflection occurs at $I_{S C L}^{o} \simeq 920$ as evident from the phase space $\left(z, u_{z}=\gamma v_{z}\right)$ plots at $I_{I N}=910$ and 925 amperes (see Figs. 4 and 5 . Thus, an opening in the drift tube lowers the space charge limited current due to an enhancement of space charge effect caused by the slowing down of electrons.

The magnitude of power loss depends on the transmitted current as also the size of the opening. Thus, the space charge limited current should decrease as the size of the opening increases. In Fig. [6] the critical current is plotted against $R_{C P}$. For smaller openings (large $R_{C P}$ ), power loss is small and thus $I_{S C L}^{o}$ is close to (but less than) $I_{S C L}^{c}(2)$. As the size of the opening increases, power loss increases thereby reducing the space charge limited current. As the opening increases further, the drop in space charge limited current reduces the field energy produced sufficiently so that power loss no longer increases as the opening is increased further. This is illustrated in Fig. 7 where the power starts reducing at smaller openings.

For completeness, we have also studied the regime with

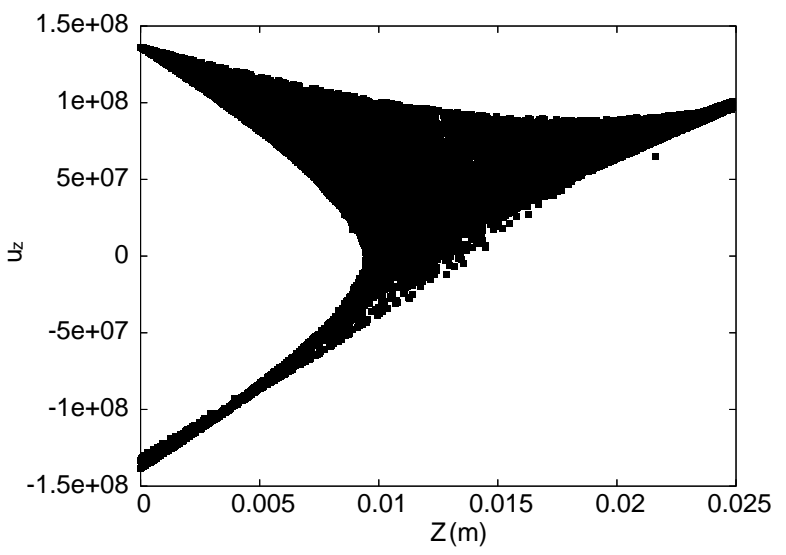

FIG. 5: The phase space for $I_{I N}=925 \mathrm{~A}$. There is reflection at this injected current.

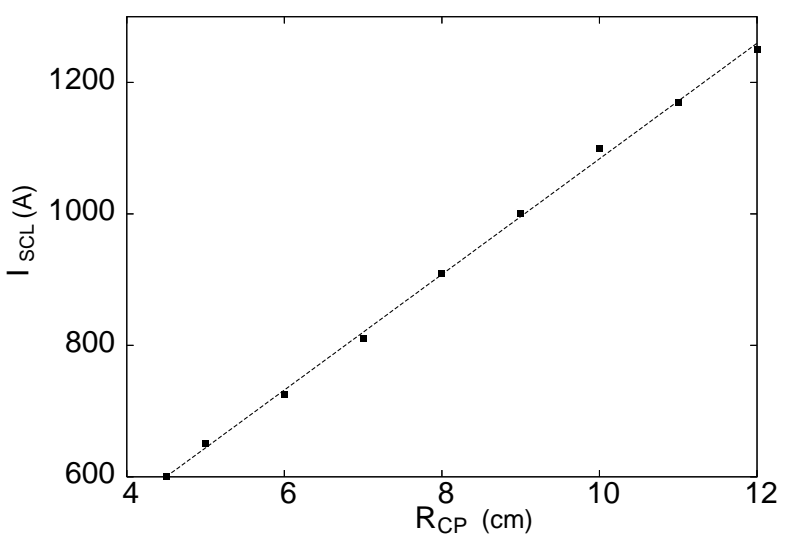

FIG. 6: The critical current as a function of the radius of the collection plate. The dashed line is the best fitting straight line

$I_{I N}>I_{S C L}^{o}$ for three different openings. As in case of a closed drift space (1-dimensional case) 2], the transmitted current drops as the injected current is increased beyond $I_{S C L}^{o}$. Note that the "classical theory" for the 1dimensional case predicts a drop in the transmitted current beyond $I_{S C L}^{c}$ and a gradual decay to $I_{S C L}^{c} / 8$ thereafter.

\section{THE EFFECTIVE VOLTAGE THEORY FOR DRIFT TUBES}

In the previous section we have seen that radiative power loss due to an opening in the drift tube leads to an equivalent drop in the electron energy reaching the collection plate. Recall that in the standard electrostatic picture 2], the emission and collection plate are at the same potential $V$ where $\mathrm{eV}$ is the kinetic energy of the injected electrons. Eq. 1 1 follows on solving Poisson equation and demanding that the potential minima be positive.

For open drift tubes, power loss can be incorporated 


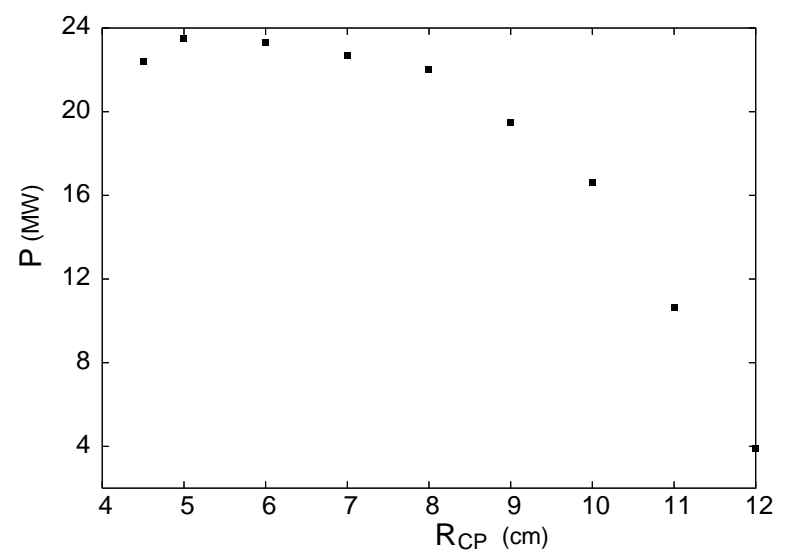

FIG. 7: Electromagnetic power emitted though the open window is plotted against $R_{C P}$.

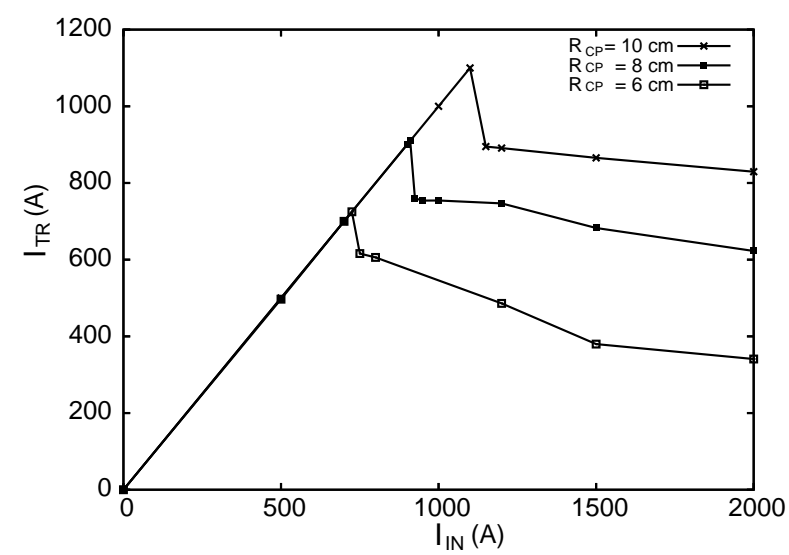

FIG. 8: The transmitted current drops as the injected current is increased beyond $I_{S C L}^{o}$. As the opening increases, the space charge limited current decreases.

within the electrostatic picture by modifying the boundary conditions appropriately. The loss of electron kinetic energy to electromagnetic radiation is equivalent to having the collection plate potential at a value $P / I_{I N}$ lower than the emission plate potential. Thus, if the emission and collection plates are at $z=0$ and $z=L$ respectively,

$$
\begin{aligned}
\phi(0) & =V \\
\phi(L) & =V-P / I_{I N}
\end{aligned}
$$

where $P$ is the electromagnetic power lost through the open window. Note that in steady state (no reflection) $I_{T R}=I_{I N}$. The space charge limited current for open drift space can thus be determined by demanding that the potential minima be positive.

Equivalently, one may study the open drift tube problem as a diode with non-zero injection energy $\mathcal{E}[9,10]$ having plate potentials

$$
\begin{aligned}
\phi(0) & =0 \\
\phi(L) & =V_{0} .
\end{aligned}
$$

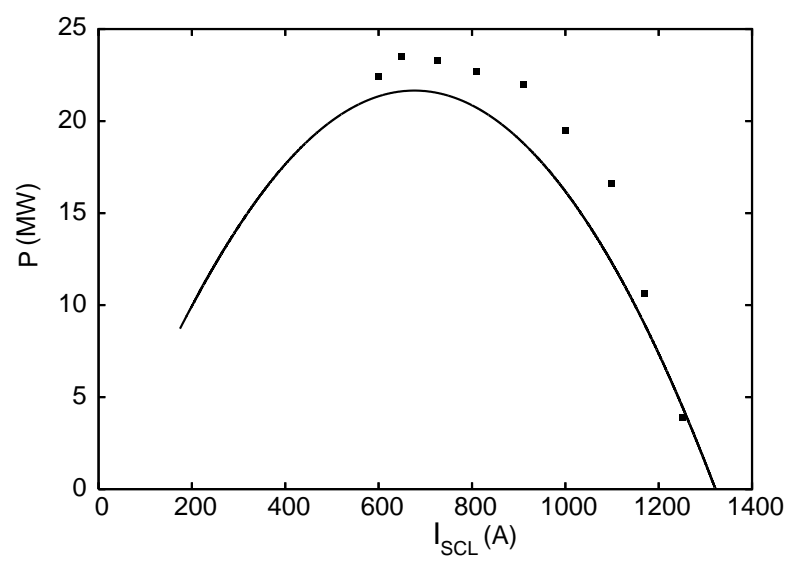

FIG. 9: The predictions of the effective voltage theory (Eq. 8 compared with PIC simulations (solid squares).

This is a well studied problem (see for instance Eq. (19) of [9]) for which the space charge limited current density is

$$
J_{S C L}^{\text {Diode }}=\frac{J_{S C L}^{c}}{2^{3}}\left(1+\sqrt{1+\frac{V_{0}}{\mathcal{E}}}\right)^{3}
$$

Applying Eq. 7 to the open drift tube problem with $V_{0}=-P / I_{I N}$, the space charge limited current in terms of the radiative power loss $P$ is:

$$
I_{S C L}^{o}=\mathcal{A} \alpha_{g} \frac{J_{S C L}^{c}}{2^{3}}\left(1+\sqrt{1-\frac{e P}{\mathcal{E} I_{S C L}^{o}}}\right)^{3}
$$

where $\mathcal{A}$ is the emission area, $\mathcal{E}$ is the injection energy and

$$
\alpha_{g}=J_{S C L}^{c}(2) / J_{S C L}^{c}
$$

is a factor that accounts for the finite geometry (for geometric factor in diodes, see [11]). In the above $J_{S C L}^{c}(2)$ is the space charge limited current in a finite closed drift tube.

Note that Eq. 8 does not directly give the space charge limited current. Instead, for each allowed power loss $P$, one must find the roots of Eq. 8 to determine the space charge limited current $I_{S C L}^{o}$. When $P=0$ (closed drift tube), $J_{S C L}^{o}=\alpha_{g} J_{S C L}^{c}=J_{S C L}^{c}(2)$. As the power loss increases, the number and values of allowed (positive) roots of Eq. 8 can be determined. However beyond a certain power loss, $P_{\max }$, there is no allowed root of Eq. 8 There is thus a maximum value of electromagnetic power loss for a given drift tube geometry and injection energy.

Fig. 9 illustrates these phases for the open drift tube under consideration. The solid line represents the roots of Eq. 8 for each value of power loss $P$ and with $\alpha_{g}=1.03$. The transmitted current is maximum when the drift tube is closed and $P=0$. Note that as $P$ increases from 0 to 
$P_{1} \simeq 8.4$, there is a single allowed value of $I_{S C L}$. This follows from Eq. 8 on noting that the expression within the square root must be positive. At $P_{1}$ for instance, the allowed values of current must exceed $I_{S C L}=168$. For $P>P_{1}$, there are two admissible roots and these correspond to the case with (i) small opening and large current and (ii) larger opening but smaller current. Beyond $P_{\max } \simeq 21.65$, there is no allowed current for the given geometry and injection energy. Fig. 9 also shows our PIC simulation results (solid squares) for values of $R_{C P}$ ranging from $4.5 \mathrm{~cm}$ to $12 \mathrm{~cm}$. The agreement with the theoretical prediction is reasonably good.

\section{DISCUSSION AND CONCLUSIONS}

In the preceding sections, we have studied the effect of electromagnetic power loss on the space charge limited current in open drift tubes. We have demonstrated using PIC simulations that even well below the space charge limited current, there is power loss which reflects in the kinetic energy of electrons reaching the collection plate. The slowing down of electrons however does not affect the transmitted current as long as the injected current is small. As the injected current increases, the power loss increases as well thereby leading to an enhancement of space charge effect. Thus the space charge limit is reached earlier in open drift tubes.

We have also shown that an estimate of the space charge limited current in open drift tubes can be obtained from electrostatic considerations by modifying the boundary conditions appropriately using an effective voltage so as to reflect the loss in energy of electrons. The predictions of this theory are in close agreement with PIC simulations.

A fallout of this theory is the existence of a limit for the electromagnetic power emanating from a given open drift tube geometry and injections energy. This can be profitably used in the design of devices used for the extraction of electromagnetic power [12].
[1] High power Microwave sources, edited by V.L. Granatstein and I. Alexeff (Artech House,Boston,1987).

[2] C.K.Birdsall and W.B.Bridges, Electron Dynamics of diode regions (Academic Press, New York, 1966).

[3] R. R. Puri, R. Kumar and D. Biswas, Theory of Vircators 1: One dimensional model (BARC Report: BARC/2002/I/022).

[4] R. B. Miller, Introduction to the Physics of Intense Charge Particle Beams (Plenum, New York, 1982).

[5] D. Biswas, R. Kumar and R. R. Puri, Phys. Plasmas 12, 093102 (2005).

[6] C. D. Child, Phys. Rev. Ser. 1 32, 492 (1911);

[7] I. Langmuir, Phys. Rev. 2, 450 (1913).
[8] J. P. Verboncoeur, A. B. Langdon and N. T. Gladd, Comp. Phys. Comm. 87, 199 (1995). The code is available at http://ptsg.eecs.berkeley.edu/\#software

[9] R. R. Puri, D. Biswas and R. Kumar Phys. Plasmas 11, 1178 (2004).

[10] P.V.Akimov, H.Schamel, H.Kolinsky, A.Ya.Ender, and V.I.Kuznetsov, Phys.Plasmas 8, 3788 (2001).

[11] J. W. Luginsland, Y. Y. Lau, R. J. Umstattd and J. J. Watrous, Phys. Plasmas 9, 2371 (2002), Y. Y. Lau, Phys. Rev. Lett. 87, 278301 (2001).

[12] D. Biswas and R. Kumar, Efficiency enhancement of the axial vircator (to be published). 